\title{
Synthesis and characterization of substituted bisbenzoxazine as potent antimicrobial agent
}

\author{
Kishor R Lalcheta and Bhvin B Dhaduk* \\ Department of Biochemistry, Saurashtra University, Rajkot, Gujarat, India
${ }^{*}$ Corresponding Author E-mail address: dhaduk.bhavin@ymail.co. \\ Bisbenoxazines derivatives were synthesized by refluxing substituted bopeno substit ced aniline \\ and formaldehyde in presence of 1, 4-dioxane with good yields. All synthesiz or pounds were \\ characterized by mass, NMR and IR and also evaluated for antimi rob. ctivity aga st four different \\ bacterial and two fungal strains. The compounds $1 \mathrm{c}, 1 \mathrm{~h}, 1 \mathrm{j}$ and $\mathrm{N}$ fou. omparatively good active
} against all the bacterial strains.

Keywords: Benzoxazines, Synthesis, Microbial activity, ectral techni ues

\section{INTRODUCTION}

Benzoxazines are six-membere terocy ic compounds synthesized via Mannich condensation reaction from $p$ enols amines, nd formaldehyde. The first benzoxazine compound was synthesized by $1 / y$ a cone 1944 [1]. Later, the study on benzoxazines and their oligomers wo rurth conducted from the 1950s to 1970s [2-4]. These benzoxazines were syp ized from osphenols, monoamines, and formaldehyde, but they could not form polyz ers th high mo ecular weight. After benzoxazines based on bisphenolA were synthesi $[5,6]$, a ries of bifunctional benzoxazines have been prepared from bisphenols (o mon whenols), sonoamines (or diamines), and formaldehyde [7-15]. The bifunctional zox anes can form into polybenzoxazines with high molecular weight via thermally acti d ring opening polymerization reaction [16-19], and the resultant polyb zin zines ss excellent properties, such as relatively high glass transition ter aratur superio mechanical properties, excellent electrical insulation properties, low mois , high thermal stability, high char yields, and low flammability. Therefore, benzox a can be used as high-performance matrices in electronics and aerospace industries. ne work on microbial screening has not been found on bisbenzoxazine derivatives. The aim of this study is to investigate the biological activity of substituted bisbenzoxazine derivatives. 


\section{EXPERIMENTAL}

All research chemicals were purchased from Allied Chemicals (Vadodara) and used as received. Thin-layer chromatography was accomplished on $0.2-\mathrm{mm}$ percolated plates of silica gel G60 F254 (Merck). Visualization was made with UV light (254 and $365 \mathrm{~nm}$ ). IR spectra were recorded on a FT-IR-8400 spectrophotometer using DRS prob. ${ }^{1} \mathrm{H}(400 \mathrm{MHz})$ and ${ }^{13} \mathrm{C}$ (400 MHz) NMR spectra were recorded on a Bruker AVANCE II spectrometer in CDCl3. Chemical shifts are expressed in $\delta$ ppm downfield from TMS as an internal standard. Mass spectra were determined using direct inlet probe on a GCMS-QP 2010 mass spectmmeter (Shimadzu). Melting points were measured in open capillaries and are uncorrecte

2. 1. General procedure for the synthesis of substituted bisbenzoxazine reriv es. (2a. ) $0.1 \mathrm{~mol}$ substituted aniline in $20 \mathrm{ml} \mathrm{1,4-dioxane} \mathrm{was} \mathrm{slowly} \mathrm{added} \mathrm{to} 0.2$ mol forma yd in $80 \mathrm{ml}$, keeping temperature below $10^{\circ} \mathrm{C}$ in an ice bath. The mixture w stirre or $10 \mathrm{C}$ and $0.05 \mathrm{~mol}$ bisphenol in $50 \mathrm{ml}$ 1, 4-dioxane was added. Temperatur of 1 xture as raised and refluxed for $15 \mathrm{~h}$, cooled, poured in the crushed ice, sepa ated yello sh so id filtered, washed well with water and dried at room temperature. Cry a o duct was ated with $3 \mathrm{~N}$ $\mathrm{NaOH}$ solution till unreacted bisphenol was removed comp tely $b$ teminent checking with a dilute $\mathrm{HCl}$ solution washed well with water and at $50^{\circ} \mathrm{C}$ ionzoxazines were recrystallized repeatedly from appropriate solvent sy em to get pure product 1(a-l).

\subsection{Biological testing}

Bisphenol-C derivatives were screened for invitro arruacterial and antifungal activities following micro broth dilution method [20-2]. gram-negative (Bacillus subtilis, Staphylococo cauren,) and gram-positive (Escherichia coli, Salmonella typhi) microorganis $n$ ntifung activity was screened against Aspergillus niger, Aspergillus clavatus m roorg isms. T c standard drugs used for this study were gentamycin, ampicillin, chora profloxacin and norfloxacin were used for antibacterial screening. ystatin a griseofulvin were used for antifungal screening. The standard strains used a reening o Mtibacterial and antifungal activities were procured from Institute of Mic obial hnology (IMTECH), Chandigarh, India. Mueller Hinton Broth was used as a ny nont mediun r pacteria and Sabouraud Dextrose Broth for fungal growth. Inoculums siz for ty strain was adjusted to $10^{8} \mathrm{CFU} / \mathrm{mL}$ by comparing the turbidity. The results were red in form of primary and secondary screening. The stock solution $(2000 \mu \mathrm{o} / \mathrm{mL}) \mathrm{o}$ ce con pounds under investigation and standard drugs were prepared by successiv ilution primary screening, 1000, 500 and $250 \mu \mathrm{g} / \mathrm{mL}$ concentrations of the cq und were used. The compounds which found active in this primary screening were furtho In secondary screening, 200, 100, 50, 25, 12.5 and $6.25 \mu \mathrm{g} / \mathrm{mL}$ concent ns, were used. The inoculated wells were incubated overnight at $37^{\circ} \mathrm{C}$ in a humid atmospher overnight. The highest dilution showing at least $99 \%$ inhibition was considered as minimum inhibition concentration (MIC).

The investigation of antibacterial and antifungal screening data revealed that all the tested compounds 4a-t showed moderate to potent activity. The compounds $4 \mathrm{i}, 4 \mathrm{k}$ and 41 showed comparatively good activity against all the bacterial strains. 


\section{RESULTS AND DISCUSSION}

\section{Chemistry}

In our current research bisbenoxazines derivatives were synthesized by refluxing substituted bisphenol-C, substituted aniline and formaldehyde in presence of 1, 4-dioxane (Scheme 1). These compounds were characterized by FTIR, ${ }^{1} \mathrm{H}$ NMR, ${ }^{13} \mathrm{C} \mathrm{NMR}$, and MS techniques.

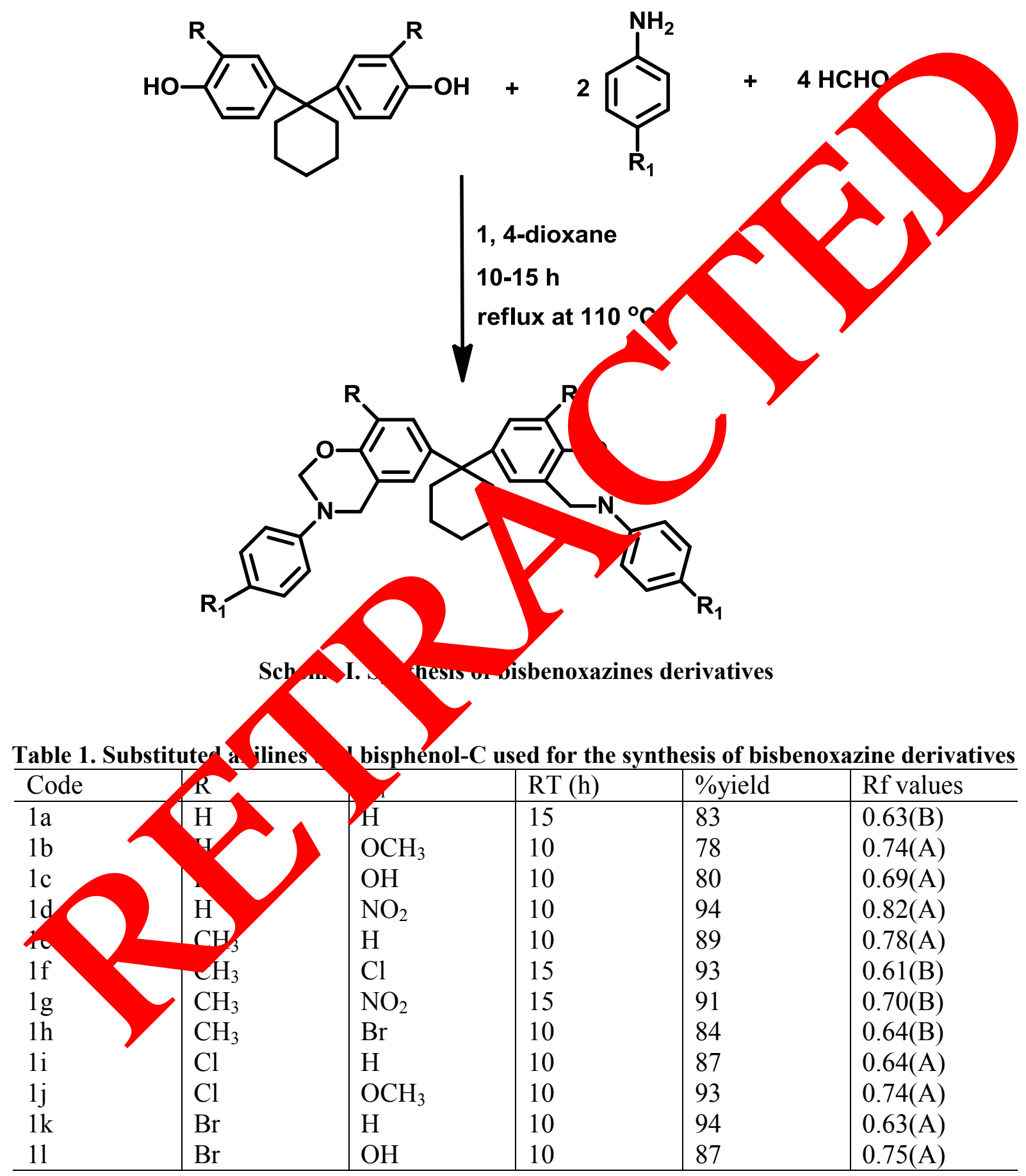




\subsection{Spectroscopic data for the compounds 1(a-l)}

6, 6'-(cyclohexane-1,1-diyl)bis(3-phenyl)-3,4-dihydro-2H-benzo[e][1,3]oxazine) (1a):

White solid, mp 185-187 ${ }^{\circ}$; IR (KBr): 3459, 3327, 3193, 2999, 1648, 1586, 1261, $1061 \mathrm{~cm}^{-1}$; ${ }^{1} \mathrm{H}$ NMR (400 MHz, Chloroform) $\delta 7.33-7.28(\mathrm{~m}, 2 \mathrm{H}), 7.27-7.09(\mathrm{~m}, 6 \mathrm{H}), 7.08-6.92(\mathrm{~m}$, 2H), $6.77-6.66(\mathrm{~m}, 6 \mathrm{H}), 6.02-5.98(\mathrm{~m}, 2 \mathrm{H}), 5.44-5.40(\mathrm{~m}, 2 \mathrm{H}), 4.85-4.81(\mathrm{~m}, 2 \mathrm{H}), 4.78$ $-4.74(\mathrm{~m}, 2 \mathrm{H}), 1.82-1.78(\mathrm{~m}, 2 \mathrm{H}), 1.72(\mathrm{t}, J=9.4 \mathrm{~Hz}, 3 \mathrm{H}), 1.46-1.36(\mathrm{~m}, 5 \mathrm{H}) ; \mathrm{MS}(\mathrm{m} / \mathrm{z})$ : $502\left(\mathrm{M}^{+}\right)$; Anal. Calcd for $\mathrm{C}_{34} \mathrm{H}_{34} \mathrm{~N}_{2} \mathrm{O}_{2}$ : C, 81.24; H, 6.82; N, 5.57; O, 6.37; Found: C, 81.20; $\mathrm{H}, 6.80 ; \mathrm{N}, 5.52 ; \mathrm{O}, 6.33$.

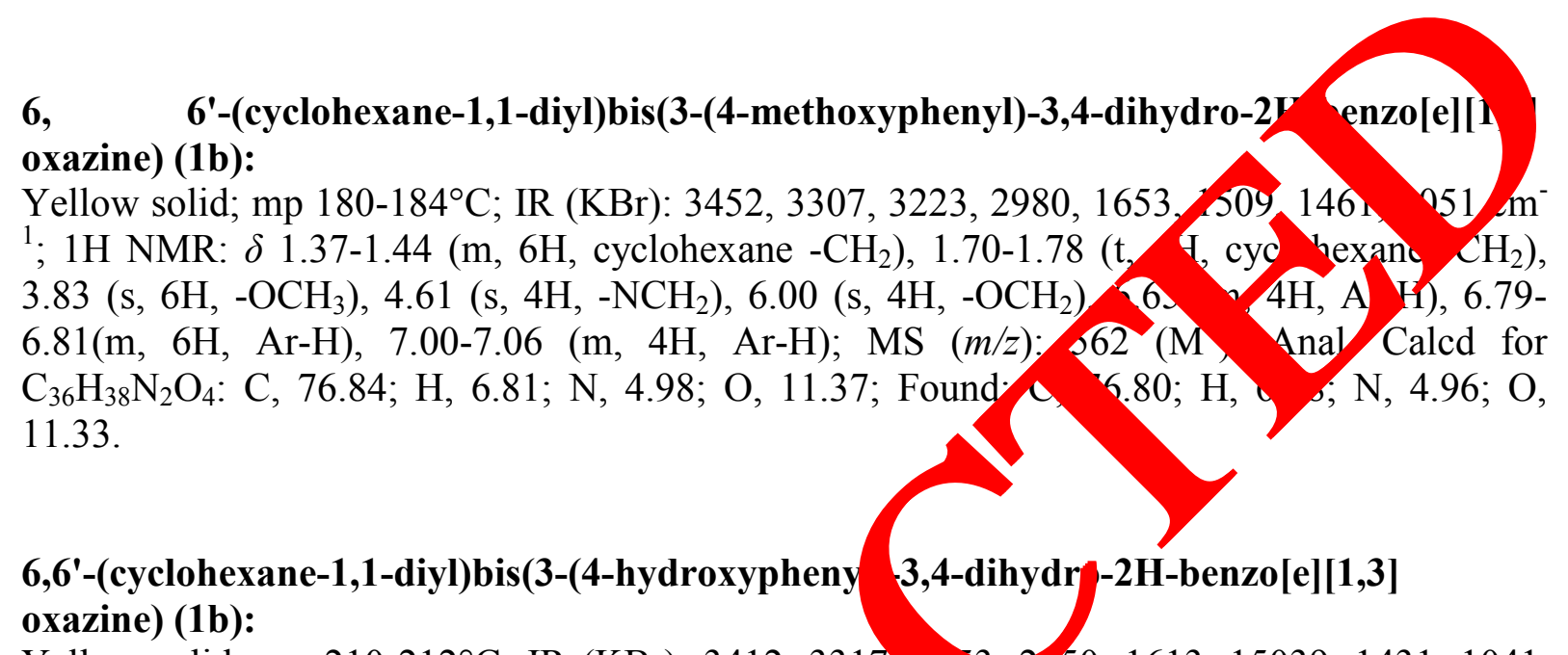

Yellow solid; mp $210-212^{\circ} \mathrm{C}$; IR (KBr): 3412, 3317, $50,1613,15039,1431,1041$ $\mathrm{cm}^{-1}$; 1H NMR: $\delta$ 1.37-1.44 (m, 6H, cyclohe on $\left.\mathrm{CH}_{2}\right), 1.70-1.78\left(\mathrm{t}, 4 \mathrm{H}\right.$, cyclohexane $\left.-\mathrm{CH}_{2}\right)$, $\left.3.83\left(\mathrm{~s}, 6 \mathrm{H},-\mathrm{OCH}_{3}\right), 4.61\left(\mathrm{~s}, 4 \mathrm{H},-\mathrm{NCH}_{2}\right), 5,5 \mathrm{~s}, \mathrm{OH}\right), 6.00\left(\mathrm{~s}, 4 \mathrm{H},-\mathrm{OCH}_{2}\right), 6.59(\mathrm{~m}$, 4H, Ar-H), 6.77-6.79 (m, 6H, Ar-H 7.00-7. (m, 4H, Ar-H); MS (m/z): $534\left(\mathrm{M}^{+}\right)$; Anal. Calcd for $\mathrm{C}_{34} \mathrm{H}_{34} \mathrm{~N}_{2} \mathrm{O}_{4}$ : C, 76.38, H, $41 ; \mathrm{N}$, . 24; O, 11.97; Found: C, 76.34; H, 6.41; N, 5.24; O, 11.95 .

6, 6'-(cyclohexan 1,1 l)bis(3-(4 nitrophenyl)-3,4-dihydro-2H-benzo[e][1,3]oxazine) (1d)

Yellow solid; $n_{1}$ 194-196 ${ }^{\circ} \mathrm{C}$; $\mathrm{NBr}$ ): $3412,3317,3253,2950,1613,1539,1431,1061 \mathrm{~cm}^{-}$ 1; $1 \mathrm{H} \mathrm{NMP} 1.3744\left(\mathrm{~m}, 6 \mathrm{H}\right.$, cyclohexane $\left.-\mathrm{CH}_{2}\right), 1.70-1.78\left(\mathrm{t}, 4 \mathrm{H}\right.$, cyclohexane $\left.-\mathrm{CH}_{2}\right)$, $3.83\left(\mathrm{~s}, 6 \mathrm{H},-\mathrm{O}, 4.61,4 \mathrm{H},-\mathrm{NCH}_{2}\right), 6.00\left(\mathrm{~s}, 4 \mathrm{H},-\mathrm{OCH}_{2}\right), 6.79(\mathrm{~d}, 2 \mathrm{H}, \mathrm{Ar}-\mathrm{H}), 7.00-7.06$ $(\mathrm{m}, 8 \mathrm{H}-\mathrm{H}), \delta \quad(\mathrm{m} / \mathrm{H}, \mathrm{Ar}-\mathrm{H}) ; \mathrm{MS}(\mathrm{m} / \mathrm{z}): 592\left(\mathrm{M}^{+}\right)$; Anal. Calcd for $\mathrm{C}_{34} \mathrm{H}_{32} \mathrm{~N}_{4} \mathrm{O}_{6}: \mathrm{C}$, $68.9 ; \mathrm{H}, \mathrm{H}+\mathrm{N}, \mathrm{N}$. $\quad \mathrm{O}, 16.20$; Found: C, 68.88; H, 5.40; N, 9.40; O, 1.20

6,6'-(cyc)
(1e) white solid; mp $194-196^{\circ} \mathrm{C}$; IR (KBr): 3449, 3331, 3182, 3055, 2952, 1651, 1568, 1491, 1247, $1049 \mathrm{~cm}^{-1}$; 1H NMR: $\delta 1.37-1.44\left(\mathrm{~m}, 6 \mathrm{H}\right.$, cyclohexane $\left.-\mathrm{CH}_{2}\right), 1.70-1.78(\mathrm{t}, 4 \mathrm{H}$, cyclohexane - $\left.\mathrm{CH}_{2}\right), 2.34\left(\mathrm{~s}, 6 \mathrm{H}, \mathrm{CH}_{3}\right), 4.61\left(\mathrm{~s}, 4 \mathrm{H},-\mathrm{NCH}_{2}\right), 5.35(\mathrm{~s}, 2 \mathrm{H},-\mathrm{OH}), 6.00(\mathrm{~s}, 4 \mathrm{H},-$ $\left.\mathrm{OCH}_{2}\right), 6.79-6.81(\mathrm{~m}, 4 \mathrm{H}, \mathrm{Ar}-\mathrm{H}), 6.94(\mathrm{~m}, 6 \mathrm{H}, \mathrm{Ar}-\mathrm{H}), 7.27$ (m, 4H, Ar-H); MS $(\mathrm{m} / \mathrm{z}): 530$ $\left(\mathrm{M}^{+}\right)$; Anal. Calcd for $\mathrm{C}_{36} \mathrm{H}_{38} \mathrm{~N}_{2} \mathrm{O}_{2}:$ C, 81.47; H, 7.22; N, 5.28; O, 6.03; Found: C, 81.40; H, 7.20; N, 5.25; O, 6.00. 
6,6'-(cyclohexane-1,1-diyl)bis(3-(4-chlorophenyl-7-methyl)-3,4-dihydro-2H benzo[e][1,3]oxazine $)(1 \mathrm{f})$

White solid; mp 245-247 ${ }^{\circ} \mathrm{C}$; $\mathrm{IR}(\mathrm{KBr})$ : 3442, 3226, 3143, 2986, 1642, 1566, 1241, $1061 \mathrm{~cm}^{-1}$; 1H NMR: $\delta$ 1.37-1.44 (m, 6H, cyclohexane $\left.-\mathrm{CH}_{2}\right), 1.70-1.78\left(\mathrm{t}, 4 \mathrm{H}\right.$, cyclohexane $\left.-\mathrm{CH}_{2}\right), 2.34$ (s, $\left.6 \mathrm{H}, \mathrm{CH}_{3}\right), 4.61\left(\mathrm{~s}, 4 \mathrm{H},-\mathrm{NCH}_{2}\right), 6.00\left(\mathrm{~s}, 4 \mathrm{H},-\mathrm{OCH}_{2}\right), 6.70(\mathrm{~d}, 4 \mathrm{H}, \mathrm{Ar}-\mathrm{H}), 6.81(\mathrm{~s}, 2 \mathrm{H}, \mathrm{Ar}-$ $\mathrm{H}), 6.94(\mathrm{~s}, 2 \mathrm{H}, \operatorname{Ar}-\mathrm{H}), 7.31(\mathrm{~d}, 4 \mathrm{H}, \operatorname{Ar}-\mathrm{H}) ; \mathrm{MS}(\mathrm{m} / \mathrm{z}): 599\left(\mathrm{M}^{+}\right)$; Anal. Calcd for $\mathrm{C}_{36} \mathrm{H}_{36} \mathrm{Cl}_{2} \mathrm{~N}_{2} \mathrm{O}_{2}$ : C, 72.11; H, 6.05; Cl, 11.83; N, 4.67; O, 5.34; Found: C, 72.09; H, 6.00; Cl, $11.80 ; \mathrm{N}, 4.63 ; \mathrm{O}, 5.30$

6,6'-(cyclohexane-1,1-diyl)bis(3-(4-nitrophenyl-7-methyl)-3,4-dihydro-2Hbenzo[e][1,3]oxazine) (1g)

White solid; mp 165-167 ${ }^{\circ}$; IR (KBr): 3462, 3327, 3220, 2980, 1623, 15 99,1461 , 1 cr ; 1H NMR: $\delta$ 1.37-1.44 (m, 6H, cyclohexane $\left.-\mathrm{CH}_{2}\right), 1.70-1.78(\mathrm{t}, 4 \mathrm{H}, 2$ lohex e - $\mathrm{CH}, 2.34$ $\left(\mathrm{s}, 6 \mathrm{H}, \mathrm{CH}_{3}\right), 4.61\left(\mathrm{~s}, 4 \mathrm{H},-\mathrm{NCH}_{2}\right), 6.00\left(\mathrm{~s}, 4 \mathrm{H},-\mathrm{OCH}_{2}\right), 6.81(\mathrm{~s}, 2 \mathrm{~T}, 6.94 \mathrm{H}, \mathrm{Ar}-$ $\mathrm{H}), 7.02(\mathrm{~d}, 4 \mathrm{H}, \mathrm{Ar}-\mathrm{H}), 8.08(\mathrm{~d}, 4 \mathrm{H}, \mathrm{Ar}-\mathrm{H})$; $\mathrm{MS}(\mathrm{m} / \mathrm{z}): 620\left(\mathrm{M}^{+}\right)$Anal. Ca for $\mathrm{C}_{36} \mathrm{H}_{36} \mathrm{~N}_{4} \mathrm{O}_{6}$ : C, 69.66; H, 5.85; N, 9.03; O, 15.47; Found: C, 69.60; H, 5.82, N, 9.00; 0.45

6,6'-(cyclohexane-1,1-diyl)bis(3-(4-bromophenyl-7 methyl)-3,4-dihydro-2Hbenzo[e][1,3]oxazine) (1h)

White solid; $\mathrm{mp} 256-258^{\circ} \mathrm{C}$; IR (KBr): 3459,3252 , 1H NMR: $\delta$ 1.37-1.44 (m, 6H, cyclohexane- $\left.\mathrm{CH}_{2}\right), 1$. $3,2919,648,1586,1241,1051 \mathrm{~cm}^{-1}$ , $4 \mathrm{H}$, cyclohexane $\left.-\mathrm{CH}_{2}\right), 2.34$ $\left(\mathrm{s}, 6 \mathrm{H}, \mathrm{CH}_{3}\right), 4.61\left(\mathrm{~s}, 4 \mathrm{H},-\mathrm{NCH}_{2}\right), 6.00\left(\mathrm{~s}, \mathrm{CH}_{2}\right), 6.65(\mathrm{~d}, 4 \mathrm{H}, \mathrm{Ar}-\mathrm{H}), 6.81(\mathrm{~s}, 2 \mathrm{H}, \mathrm{Ar}-$ $\mathrm{H}), 6.94(\mathrm{~s}, 2 \mathrm{H}, \mathrm{Ar}-\mathrm{H}), 7.42(\mathrm{~d}, 4 \mathrm{H}, \mathrm{A} \mathrm{H}) \cdot \mathrm{m} / \mathrm{z}): 688\left(\mathrm{M}^{+}\right)$; Anal. Calcd for $\mathrm{C}_{36} \mathrm{H}_{36} \mathrm{Br}_{2} \mathrm{~N}_{2} \mathrm{O}_{2}$ : C, 62.80; H, 5.27; $\mathrm{Rr}$ 23.21; 4.07; O, 4.65; Found: C, 62.78; H, 5.25; Br, $23.18 ; \mathrm{N}, 4.03 ; \mathrm{O}, 4.02$

6,6'-(cyclohexane-1,1-d' 'T)bis(3-ph

(1i)

White solid; mp 1 $5-67^{\circ} \mathrm{C},(\mathrm{KBr}): 3462,3327,3220,2980,1623,1509,1461,1051 \mathrm{~cm}^{-1}$; 1H NMR: $\delta 1.3 / 1.44\left(\mathrm{~m}, 6 \mathrm{H}\right.$, Mohexane $\left.-\mathrm{CH}_{2}\right), 1.70-1.78\left(\mathrm{t}, 4 \mathrm{H}\right.$, cyclohexane $\left.-\mathrm{CH}_{2}\right), 2.34$ $\left.\left(\mathrm{s}, 6 \mathrm{H}, \mathrm{CH}_{3}\right) \quad 61(\mathrm{~s}) \mathrm{H}, \mathrm{NCH}_{2}\right), 6.00\left(\mathrm{~s}, 4 \mathrm{H},-\mathrm{OCH}_{2}\right), 6.79(\mathrm{~m}, 2 \mathrm{H}, \mathrm{Ar}-\mathrm{H}), 6.94(\mathrm{~m}, 4 \mathrm{H}, \mathrm{Ar}-$ $\mathrm{H}), 6.99-7.00 \quad 4 \mathrm{H}, \mathrm{Ar} \mathrm{H}), 7.27(\mathrm{~m}, 4 \mathrm{H}, \mathrm{Ar}-\mathrm{H})$; $\mathrm{MS}(\mathrm{m} / \mathrm{z}): 571\left(\mathrm{M}^{+}\right)$; Anal. Calcd for $\mathrm{C}_{34} \mathrm{H}_{3}{ }_{2} \mathrm{O}_{2}: \quad .45, \mathrm{H}, 5.64 ; \mathrm{Cl}, 12.41 ; \mathrm{N}, 4.90, \mathrm{O}, 5.60 ;$ Found: $\mathrm{C}, 71.45 ; \mathrm{H}, 5.64 ; \mathrm{Cl}$, $12.4 ; \mathrm{N}, 2 ; 5 ; \mathrm{O}, 5$.

6,6'-(cych yane-1,1-diyl)bis(3-(4-methoxypheny7-chloro)-3,4-dihydro-2Hbenzo[e][1,,3]oxazine) $(1 \mathrm{j})$

White solid; $\mathrm{mp} 178-180^{\circ} \mathrm{C}$; IR (KBr): 3442, 3327, 3173, 2989, 1653, 1586, 1261, $1061 \mathrm{~cm}^{-1}$; 1H NMR: $\delta$ 1.37-1.44 (m, 6H, cyclohexane $\left.-\mathrm{CH}_{2}\right), 1.70-1.78\left(\mathrm{t}, 4 \mathrm{H}\right.$, cyclohexane $\left.-\mathrm{CH}_{2}\right), 2.34$ $\left(\mathrm{s}, 6 \mathrm{H}, \mathrm{CH}_{3}\right), 4.61\left(\mathrm{~s}, 4 \mathrm{H},-\mathrm{NCH}_{2}\right), 6.00\left(\mathrm{~s}, 4 \mathrm{H},-\mathrm{OCH}_{2}\right), 6.79(\mathrm{~m}, 2 \mathrm{H}, \mathrm{Ar}-\mathrm{H}), 6.94(\mathrm{~m}, 4 \mathrm{H}, \mathrm{Ar}-$ $\mathrm{H})$, 6.99-7.00 (m, 4H, Ar-H), $7.27(\mathrm{~m}, 4 \mathrm{H}, \operatorname{Ar}-\mathrm{H})$; MS $(\mathrm{m} / \mathrm{z}): 631\left(\mathrm{M}^{+}\right)$; Anal. Calcd for $\mathrm{C}_{36} \mathrm{H}_{36} \mathrm{Cl}_{2} \mathrm{~N}_{2} \mathrm{O}_{4}$ : C, 68.46; H, 5.75; Cl, 11.23; N, 4.44; O, 10.13; Found: C, 68.40; H, 5.70; $\mathrm{Cl}$, 11.20, N, 4.40, O, 10.09 . 


\section{6,6'-(cyclohexane-1,1-diyl)bis(3-phenyl-7-bromo)-3,4-dihydro-2H-benzo[e][1,3]oxazine)}

(1k)

White solid; $\mathrm{mp} 240-242^{\circ} \mathrm{C}$; IR (KBr): 3420, 3226, 3143, 2988, 1632, 1546, 1231, $1061 \mathrm{~cm}^{-1}$; 1H NMR: $\delta$ 1.37-1.44 (m, 6H, cyclohexane $\left.-\mathrm{CH}_{2}\right), 1.70-1.78\left(\mathrm{t}, 4 \mathrm{H}\right.$, cyclohexane $\left.-\mathrm{CH}_{2}\right), 2.34$ $\left(\mathrm{s}, 6 \mathrm{H}, \mathrm{CH}_{3}\right), 4.61\left(\mathrm{~s}, 4 \mathrm{H},-\mathrm{NCH}_{2}\right), 6.00\left(\mathrm{~s}, 4 \mathrm{H},-\mathrm{OCH}_{2}\right), 6.79(\mathrm{~m}, 2 \mathrm{H}, \mathrm{Ar}-\mathrm{H}), 6.94-6.95(\mathrm{~m}$, 4H, Ar-H), 7.04 (s, 2H, Ar-H), $7.27(\mathrm{~m}, 4 \mathrm{H}, \operatorname{Ar}-\mathrm{H})$; MS $(\mathrm{m} / \mathrm{z}): 660\left(\mathrm{M}^{+}\right)$; Anal. Calcd for $\mathrm{C}_{34} \mathrm{H}_{32} \mathrm{Br}_{2} \mathrm{~N}_{2} \mathrm{O}_{2}$ : C, 61.83; H, 4.88; Br, 24.20; N, 4.24; O, 4.85; Found: C, 61.79; H, 4.84; Br, 24.15; N, 4.20; O, 4.80.

6,6'-(cyclohexane-1,1-diyl)bis(3-(4-hydroxyphenyl-7-bromo)-3,4-dihydro-2V benzo[e][1,3]oxazine) (1I)

White solid; mp 194-196 C; IR (KBr): 3412, 3317, 3253, 2950, 1613, 15?, 431 , $61 \mathrm{~cm}$; 1H NMR: $\delta 1.37-1.44\left(\mathrm{~m}, 6 \mathrm{H}\right.$, cyclohexane $\left.-\mathrm{CH}_{2}\right), 1.70-1.78(\mathrm{t}, 4 \mathrm{H}$, cy clohed ne -C 2.34 $\left(\mathrm{s}, 6 \mathrm{H}, \mathrm{CH}_{3}\right), 4.61\left(\mathrm{~s}, 4 \mathrm{H},-\mathrm{NCH}_{2}\right), 5.35(\mathrm{~s}, 2 \mathrm{H},-\mathrm{OH}), 6.00\left(\mathrm{~s}, 4 \mathrm{H},-\mathrm{H}_{2}\right)$ o.s ( $4 . \mathrm{A}, \mathrm{Ar}-$ $\mathrm{H}), 6.77$ (d, 4H, Ar-H), 6.95 (s, 2H, Ar-H), 7.04 (s, 2H, Ar-H) ivn (m $692\left(\Lambda^{\circ}\right)$; Anal. Calcd for $\mathrm{C}_{34} \mathrm{H}_{32} \mathrm{Br}_{2} \mathrm{~N}_{2} \mathrm{O}_{4}$ : C, 58.97; H, 4.66; Br, 23.08; N, 4.0 P. 9.24; $\mathrm{F}$ d. C, 58.94; H, 4.60; $\mathrm{Br}, 23.08 ; \mathrm{N}, 4.05 ; \mathrm{O}, 9.20$.

Table 2 Microbial activity of 1 phenol-C de vatives

\begin{tabular}{|c|c|c|c|c|c|c|}
\hline \multirow[t]{3}{*}{ Compound } & \multicolumn{4}{|c|}{ Antibacterial acti } & \multicolumn{2}{|c|}{ Antifungal activity } \\
\hline & E. coli & S. typhi & & reus & A. niger & A. clavattus \\
\hline & $\begin{array}{c}\text { MTCC } \\
443\end{array}$ & & 41 & $\begin{array}{c}\text { MTCC } \\
96\end{array}$ & $\begin{array}{c}\text { MTCC } \\
282\end{array}$ & $\begin{array}{c}\text { MTCC } \\
1323\end{array}$ \\
\hline $1 \mathrm{a}$ & 125 & & 200 & 250 & 1000 & 250 \\
\hline $1 \mathrm{~b}$ & 200 & & 250 & 200 & $>1000$ & 500 \\
\hline $1 \mathrm{c}$ & & & 125 & 200 & 500 & 500 \\
\hline $1 d$ & & 250 & 500 & 500 & 500 & $>1000$ \\
\hline $1 \mathrm{e}$ & & 200 & 500 & 250 & 1000 & $>1000$ \\
\hline 1f & 125 & 250 & 250 & 500 & 500 & $>1000$ \\
\hline & 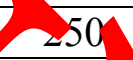 & 250 & 200 & 250 & $>1000$ & 500 \\
\hline & 100 & 200 & 62.5 & 250 & 100 & 500 \\
\hline & & 500 & 100 & 100 & 250 & $>1000$ \\
\hline & 62.5 & 125 & 250 & 200 & 250 & $>1000$ \\
\hline & 250 & 125 & 125 & 100 & $>1000$ & $>1000$ \\
\hline & 62.5 & 250 & 200 & 200 & 1000 & 1000 \\
\hline Gentamy 1 in & 0.05 & 5 & 1 & 0.25 & - & - \\
\hline Ampicillin & 100 & 100 & 250 & 250 & - & - \\
\hline Chloramphenicol & 50 & 50 & 50 & 50 & - & - \\
\hline Ciprofloxacin & 25 & 25 & 50 & 50 & - & - \\
\hline Norfloxacin & 10 & 10 & 100 & 10 & - & - \\
\hline Nystatin & - & - & - & - & 100 & 100 \\
\hline Griseofulvin & - & - & - & - & 100 & 100 \\
\hline
\end{tabular}


Bold digits indicate that synthesized compounds are comparatively active as standard drugs DMF used as control and its antibacterial activity is nil or zero

\section{CONCLUSION}

In summary, we have described the synthesis of substituted bisbenzoxazines derivatives in excellent yields by simple refluxing. The spectral data are incorporate with the structure of compounds 1a-1. The antimicrobial data reported here which may be the better refermae for the future research in the class of pyrimidine derivatives.

\section{Acknowledgement}

Author thankful to Department of Biochemistry, Saurashtra University for providir cilitie d SAIt sunjab University for instrumental support.

\section{References}

[1] Holly F.W, Cope A. C., J Am Chem Soc. 66 (194 1875.

[2] Burke W.J, Weatherbee C., J Am Chem Soc. 72 50) 4691

[3] Burke W.J, Stephens C.W. J Am Chem T4(1952) 1518.

[4] Burke W.J, Murdoch K.C, Ec. G., J Am C Soc./6 (1954) 1677.

[5] Ning X, Ishida H., J Polym ci Par A. 32 (1 4) 1121.

[6] Ning X, Ishida H., J P Aym Sc. t B. 52 (1994) 921.

[7] Liu Y.F, Yue Z.Q Gau G., Polym-r. 51 (2010) 3722.

[8] Jia K, Xu N.L, Zhao R, Liu, B, Polym Int. 60 (2011) 414.

[9] Chen K.C, T, Che W.B, Liao C.H, Sun K.W, Chang F.C., Polym Int. 60 (2011)436.

[10] 11 S. Wang \& Appl Polym Sci. 99 (2006) 1359.

[11] L .rong S.L, Hsieh C.W, Lee H.H., Polymer. 49 (2008) 1220.

[12] Spony in M, Larrechi M.S, Ronda J.C, Galia 'M, Ca'diz V., J Polym Sci Part A. 46 (2008) 7162 .

[13] Andronescu C, Gârea S.A, Deleanu C, Iovu H., Thermochim Acta. 530 (2012) 42.

[14] Chozhan C.K, Alagar M, Gnanasundaram P., Acta Mater. 57 (2009) 782.

[15] Chang S.L, Lin C.H. Facile., J Polym Sci Part A. 48 (2010) 2430. 
[16] Ishida H, Rodriguez Y., Polymer. 36 (1995) 3151.

[17] Russell V.M, Koenig J.L, Low H.Y, Ishida H., J Appl Polym Sci. 70 (1998) 1413.

[18] Wang YX, Ishida H., Polymer. 40 (1999) 4563.

[19] Wang YX, Ishida H., Macromolecules. 33 (2000) 2839.

[20] National committee for clinical laboratory standards.,5th ed.; NCCLS: Wayne, PA, 2000.

[21] Patel, N. B.; Shaikh, A. R., Indian J. Chem. 49 (2010) 929.

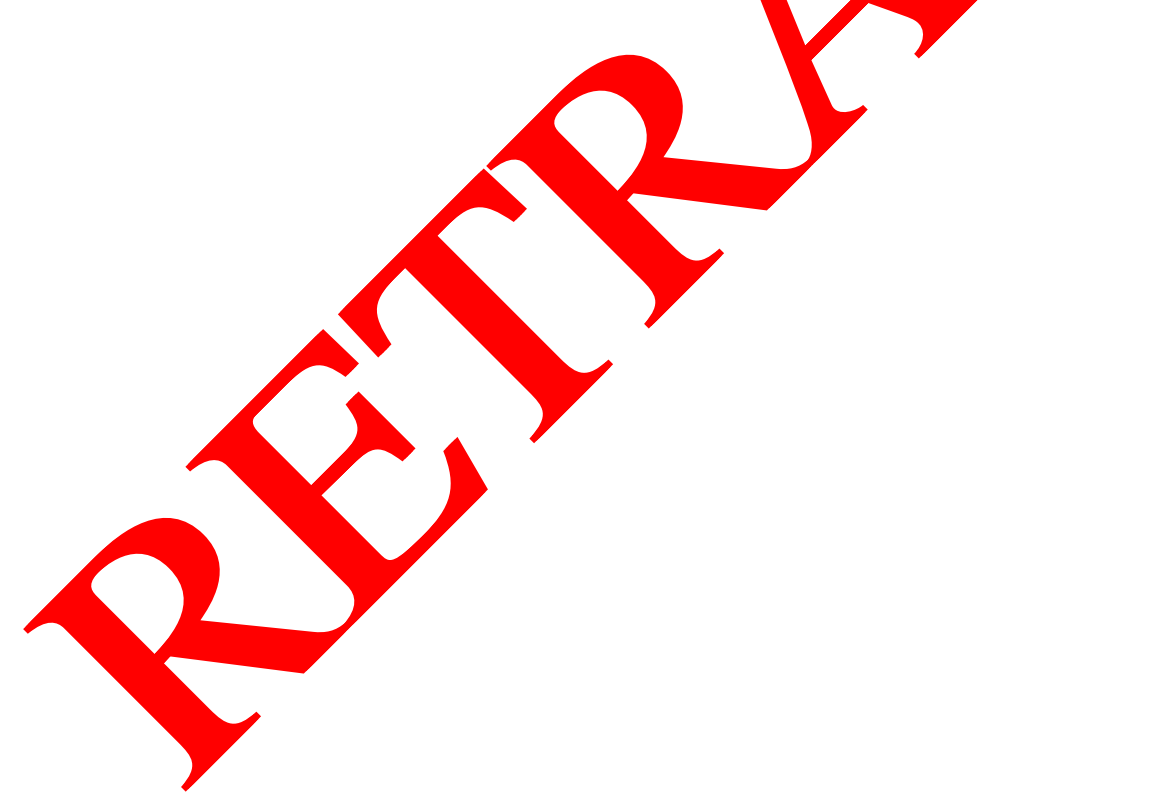

